\title{
CrimRxiv
}

\section{Risk factors associated with driving after cannabis use among Canadian young adults}

Huỳnh, Christophe, Beaulieu-Thibodeau, Alexis, Fallu, Jean-Sébastien, Bergeron, Jacques, Flores-Aranda, Jorge, Jacques, Alain, Brochu, Serge

Published on: Mar 03, 2022

DOI: $10.21428 / c b 6 a b 371 . f 273 c f 9 c$

License: Creative Commons Attribution 4.0 International License (CC-BY 4.0). 
